\title{
Role of Highly Sensitive C- Reactive, Protein, Interleukin-6 and Procalcitonin in Early Diagnosis of Neonatal Sepsis
}

\author{
Parul Singhal*, Vichal Rastogi and Ayesha Nazar
}

Department of Microbiology, School of Medical Sciences and Research, Sharda University, Greater Noida-201306, Uttar Pradesh, India

*Corresponding author

\section{Keywords}

Blood culture,

Neonatal sepsis, EONS (Early onset neonatal sepsis), LONS (Late onset neonatal sepsis), Hs-CRP (Highly Sensitive C-reactive protein), (PCT) Procalcitonin, IL-6 (Interleukin-6)

Article Info

Accepted:

04 March 2019

Available Online:

10 April 2019

\section{A B S T R A C T}

The diagnosis of neonatal sepsis is difficult because clinical manifestation is non-specific. Timely detection and treatment will help in decreasing mortality and morbidity. Blood culture is gold standard but the diagnosis is often retrospective. Thus there is a need for an alternative method which is more rapid, reliable and sensitive. This study was designed to determine the sensitivity, specificity, NPV, PPV and accuracy of hs-CRP, IL-6 and PCT levels, as a diagnostic marker of EONS. 206 neonates with sign and symptoms of sepsis admitted in NICU were included in this study. Blood culture was performed by Bac T/Alert 3D method, hs-CRP, IL-6 and PCT by ELISA. Low birth weight and prematurity were the most common risk factors present in $74 \%$ of EOS and $66 \%$ of LOS cases. Prolonged IV antibiotics were a risk factor in $26.4 \%$ in EOS and $50 \%$ in LOS. CoNS $(44.1 \%)$ was the predominant bacterial pathogen isolated, followed by Staphylococcus aureus (11.7\%), Escherichia coli (11.7\%), Acinetobacter baumanii (8.8\%) and Klebsiella pneumoniae $(8.8 \%)$ in EOS cases. Cut off value of $5 \mathrm{mg} / \mathrm{ml}$ for hs-CRP, $<20 \mathrm{pg} / \mathrm{ml}$ for IL6 and $<12 \mathrm{pg} / \mathrm{ml}$ for PCT were taken as negative. PCT is more sensitive and specific in EOS than IL-6 followed by hs-CRP. Both PCT and IL-6 are equally good markers, if patient presented later.

\section{Introduction}

According to National Neonatal Perinatal Database from hospitals, the incidence ranges from $0.1 \%$ to $4.5 \%$ with an overall mortality rate varying from $22-30 \%$. In the Western literature, the incidence of neonatal sepsis ranges from 0.01 to 0.8 percent, although early onset and late onset septicemia have different incidences but other factors such as gestational age play a major role in the incidence of septicemia, which increases with decreasing gestational age [Stoll B.J., Hansen et al., 2010 Murphy et al., 2012). In addition the case fatality rate for early and late onset sepsis has been reported as high as $40 \%$ and 19.7\% respectively (Motara et al., 2005). According to report of the WHO, about 5 million deaths are caused annually by neonatal septicemia (Kayange et al., 2010) 
National Neonatology Forum of India defines neonatal sepsis as follows (National Neonatology Forum of India, 2002):

\section{Proven sepsis}

The baby presents with clinical picture of sepsis and isolation of pathogens from blood, C.S.F., Urine or other body fluids or autopsy evidence of sepsis. Probable Sepsis: Newborn with clinical picture suggestive of sepsis with one or more of the following criteria: 1 . Existence of predisposing factors e.g., maternal fever, foul smelling liquor or prolonged rupture of the membrane (> 12 hours) or gastric polymorphs more than 6/high power field. 2. Positive septic screen (Two of the four parameters to be present) Total leucocytes count $<5,000 / \mathrm{mm}^{3}$, immature to total neutrophil count ratio $>0.2$, C-reactive protein positive and micro ESR > $15 \mathrm{~mm} /$ Ist hour or > age in days + 3. 3 . Radiological evidence of pneumonia.

Escherichia coli, Group B Streptococcus (GBS), Coagulase-negative Staphylococcus (CoNS), Haemophilus influenza and Listeria monocytogenes happen to be most common organisms (Stoll BJ, 2005, Abdollah A 2012, van den Hoogen A 2009) and In contrast, late onset sepsis (>72 hours-28 days) LOS is most commonly caused by CoNS, Staphylococcus aureus, E. coli, Klebsiella and Pseudomonas spp (Stoll, 2005; Abdollah, 2012; van den Hoogen, 2009; Sharma, 1987).

The common organisms reported by NNPD (National Neonatal Perinatal Database) Survey showed 3.8\% incidence of neonatal sepsis from pooled hospital data with Klebsiella, Staph aureus, E. coli, Pseudomonas, Enterobacter, Coagulase negative Staphylococcus, Acinetobacter and candida as the predominant organisms. Group B Streptococcus, which is a common etiological agent of early onset sepsis in
Western countries is rarely encountered in Indian scenario (Chugh, 1988 and Chaturvedi, 1989).

The manifestation of sepsis depends on whether it is 'early onset' or 'late onset' and association of serious bacterial infection e.g., meningitis, septicemia, pneumonia, bone and joint infection, urinary tract infection and necrotizing enterocolitis.

Isolation of microorganism from body fluids such as blood, CSF and urine remains the gold standard method for diagnosis of neonatal infection, but microbiological culture is not available before at least $36-48 \mathrm{hrs}$ (Fanaroff and MacDonald, 2006). In order to prevent microbial resistance induced by unnecessary administration of antibiotics, a definite diagnosis should be made using laboratory test with higher diagnostic value (Alireza and Shahian, 2007).

The commonly used markers are TNF-alfa (Tumor necrosis factor-alfa), IL-6 (Interleukin-6), IL-8 (interleukin-8), CRP (creactive protein), hs CRP (highly sensitive Creactive protein) and PCT (procalcitonin). Procalcitonin concentrations increase within 2 to 4 hours, after an exposure, peaks at 6 to 8 hours and remains elevated for the next 24 hours. Procalcitonin is secreted mainly by the neuroendocrine cells of the lungs and intestine in response to bacterial endotoxin or inflammatory cytokines (TNF, IL-6) (Maruna et al., 2000).

It does not rise significantly with viral or noninfectious inflammations, hence it is highly specific to bacterial sepsis. Furthermore, PCT is released into the circulation within 3 hours of onset of infection, plateaus at 6 hours, and remains elevated for 24 hours. This makes PCT a promising new agent for early and sensitive identification of infected neonates (José B 
López Sastre et al., 2007). Interleukin 6 (IL6 ), a chemokine produced by the $\mathrm{T}$ and $\mathrm{B}$ lymphocytes. Its level increases 2 days before clinical diagnosis of sepsis. It is more sensitive than CRP, but it cannot be used as a sole marker of sepsis, as it has a short half life. CRP is a rapidly responsive acute phase reactant, which is synthesized by the liver within 6-8 hours of stimulus of inflammatory process. Highly sensitive CRP (hs-CRP) is more sensitive than the conventional CRP, hsCRP assays measures the CRP levels lower than that measured by the conventional CRP assays because newborns cannot produce sufficient amount of acute phase proteins and so they respond to infection with a smaller increase in CRP. It's measurement below $1 \mathrm{mg} / \mathrm{l}$ provides increased sensitivity for neonatal infection (Edgar DM, Gabriel, 2002]. This test is often requested to help discriminate viral infections from bacterial infections or monitor the response to antibiotics.

Finding a reliable laboratory test as a marker for immediate detection of infection with acceptable sensitivity and specificity has always been controversial among investigators, no single test is of high diagnostic value. Therefore, a combination of at least 2 or more tests, if abnormal, leads to an increase in predictability for early diagnosis of neonatal sepsis. In previous studies IL-6, PCT and hs-CRP were not evaluated simultaneously. This is an attempt done in a Microbiology Department of Sharda University to evaluate the serum levels of IL6, PCT and hs-CRP as early diagnostic markers in neonatal infection.

\section{Materials and Methods}

The prospective study was conducted on neonates admitted for sepsis workup in the Neonatal ICU of Sharda Hospital, Greater Noida from January 2017 to December 2017.
This study was conducted in the Department of Microbiology at Sharda Hospital, Greater Noida. This study was approved by the Institutional Scientific and Ethical Committee, and written informed consents were obtained from the parents.

A total of 206 neonates with suspected sepsis who required sepsis evaluation were considered. The inclusion criteria were neonates who were admitted to the NICU with clinical signs suggestive of sepsis. The exclusion criteria were infants who were on antibiotics or those who had congenital anomalies. According to WHO IMNCI guidelines possible serious bacterial infection include following criteria: convulsions or fast breathing (60 breaths per minute or more) or nasal flaring or grunting or bulging fontanelle or 10 or more skin pustules or a big boil or axillary temperature $37.5^{\circ} \mathrm{C}$ (or feels hot to touch) or temperature less than $35.5^{\circ} \mathrm{C}$ (or feels cold to touch) or lethargy or unconsciousness or less than normal movement.

\section{Blood culture}

Blood culture was performed by automated blood culture system in all the cases. Approximately $1 \mathrm{ml}$ of blood was inoculated aseptically into BacT/Alert pediatric blood culture bottle. BacT/Alert bottles were incubated in BacT/Alert 3D system for 7 days. Growth obtained was identified by standard microbiological techniques. Identification of the organisms was based on cultural characteristics, results of various tests and biochemicals.

\section{PCT, IL-6, CRP assay}

Blood samples were centrifuged within 30 minutes of collection. Serum was stored at 20 degree Celsius before analysis. Manufacturer's instructions were followed during testing and interpretation of the three 
serological acute phase markers. PCT levels were estimated by a quantitative ELISA kit (Boster Human Procalcitonin (PCT) ELISA Kit). IL-6 levels by a quantitative ELISA kit (Boster IL6 ELISA kit) in serum and a solid phased enzyme immunoassay for the quantitative determination of hs-CRP in serum.

According to time of onset of clinical symptoms of sepsis, neonates were classified into two categories of infection as follows: (a) Group I Early onset sepsis (EOS): $<72$ hours (b) Group II Late onset sepsis (LOS): (>72 hours-28 days)

\section{Data collection and management}

Self-designed, pre tested proforma was used to collect demographic data, clinical presentation, associated risk factors (maternal $\&$ neonatal), results of the laboratory investigation generated during the admission.

\section{Statistical analysis}

All the statistical data analysis was done with the help of the SPSS version 22. For qualitative and quantitative data, Chi square test was done to analyze the data. $p$ value less than or equal to 0.05 was considered to be statistically significant.

\section{Results and Discussion}

A total of 206 neonates with sign and symptoms of sepsis admitted in NICU of Sharda Hospital were included in this study with the aim to study the role of hs- CRP, IL6 and PCT in the diagnosis of neonatal sepsis in a tertiary care hospital and to correlate the findings of quantitative hs-CRP, IL-6 and PCT with Blood culture in cases of neonatal bacterial sepsis.

Out of 206 clinically suspected cases of neonatal sepsis screened, $94(45.6 \%)$ cases were blood culture positive and remaining $112(54.3 \%)$ were blood culture negative. Pure bacterial growth was obtained from 46/206 (22.3\%) cases.

Out of the total 46 bacterial culture positive cases of sepsis, 34/46 (73.91\%) cases were in the age group ( $<72$ hours) known as Early onset sepsis (EOS) and $12(26.08 \%)$ cases were in the group (>72 hours-28 days) known as Late onset sepsis (LOS).

Lethargy and feed intolerance were the most common clinical presentation in both EOS and LOS, present in almost $100 \%$ of cases followed by respiratory distress in $78 \%$ and $50 \%$ cases of EOS and LOS respectively

\section{Risk factors}

Distribution of various risk factors associated with EOS and LOS in neonates is shown in Table 1 and 2.

Low birth weight and prematurity were the most common risk factors present in $74 \%$ of EOS and $66 \%$ of LOS cases. Prolonged IV antibiotics were a risk factor in $26.4 \%$ in EOS and $50 \%$ in LOS and ventilator support were less common risk factors observed.

\section{Isolation of different bacterial species}

Coagulase negative Staphylococcci (44.1\%) was the predominant bacterial pathogen isolated, followed by Staphylococcus aureus (11.7\%), Escherichia coli (11.7\%), Acinetobacter baumanii (8.8\%) and Klebseilla Pneumoniae (8.8\%) in EOS cases (Table 3).

However, Coagulase negative Staphylococcci $(41.6 \%)$ was the predominant bacterial isolate in LOS cases, followed by Staphylococcus aureus (16.6\%) and Acinetobacter lowffi $(8.8 \%)$ (Table 4). 
A total of 206 cases of suspected neonatal septicemia were selected on the basis of clinical presentations and risk factors associated with it. Blood culture was positive in $45.6 \%$ cases. Pure bacterial growth was obtained in $22.3 \%$ of cases.

A total of $74 \%$ cases were of EOS and $26 \%$ were of LOS. Lethargy and feed intolerance were the most common clinical presentation in both EOS and LOS, present in almost $100 \%$ of cases by respiratory distress in $78 \%$ and $57 \%$ of cases of EOS and LOS cases respectively. Bleeding tendency, convulsions and poor perfusion were the other less common clinical presentations. Low birth weight and prematurity were the most common risk factors present in $74 \%$ of EOS and $66 \%$ of LOS cases.
Coagulase Negative Staphylococci were the predominant bacterial pathogen isolated in both EOS and LOS (44\% and $41 \%$ respectively). Staphylococcus aureus (11.7\%), Escherichia coli (11.8\%), Acinetobacter baumannii (8.8\%) and Klebsiella pneumonia (8.8\%) were other pathogen isolated in EOS and Acinetobacter lwoffi (16.6\%), Staphylococcus aureus (6.4\%) and Enterococcus species (8.3\%) were isolated from cases of LOS.

In EOS Sensitivity, Specificity, PPV, NPV and Accuracy for PCT was $82.35 \%, 77.5 \%$, $75.6 \%, 83.18 \%$ and $79.72 \%$. For IL-6 was $76.47 \%, 70 \%, 68.4 \%, 77.7 \%$ and $72.97 \%$ respectively and for hs-CRP it was Sensitivity $70.5 \%$, Specificity 60\%, PPV 60\%, NPV $70.5 \%$ and Accuracy $64.86 \%$.

Table.1 Distribution of various risk factors identified in cases of EOS $(n=34)$

\begin{tabular}{|l|c|c|}
\hline Risk Factors & No. of cases & Percentage \% \\
\hline Low birth weight & 25 & $74.51 \%$ \\
\hline Prematurity & 25 & $74.51 \%$ \\
\hline Prolonged IV antibiotics & 9 & $26.4 \%$ \\
\hline Ventilator Support & 3 & $10 \%$ \\
\hline
\end{tabular}

Table.2 Distribution of various risk factors identified in cases of $\operatorname{LOS}(n=12)$

\begin{tabular}{|l|c|c|}
\hline Risk Factors & No. of cases & Percentage \% \\
\hline Low birth weight & 8 & 66.6 \\
\hline $\begin{array}{c}\text { Prematurity } \\
\begin{array}{c}\text { Prolonged } \\
\text { antibiotics }\end{array}\end{array}$ & 8 & 66.6 \\
\hline $\begin{array}{c}\text { IV } \\
\text { Ventilator support }\end{array}$ & 6 & 50 \\
\hline
\end{tabular}


Table.3 Various bacterial pathogen isolated from blood culture in cases of EOS $(n=34)$

\begin{tabular}{|l|l|l|}
\hline & Number & $\%$ \\
\hline Coagulase Negative Staphylococci & 15 & 44.1 \\
\hline Staphylococcus aureus & 4 & 11.7 \\
\hline Escherichia coli & 4 & 11.7 \\
\hline Acinetobacter baumannii & 3 & 8.8 \\
\hline Streptococcus viridians & 1 & 2.9 \\
\hline Enterococcus faecium & 1 & 2.9 \\
\hline Klebsiella pneumonia & 3 & 8.8 \\
\hline Citrobacter koseri & 2 & 2.9 \\
\hline Pseudomonas species & 1 & 5.8 \\
\hline
\end{tabular}

Table.4 Various bacterial pathogen isolated from blood culture in cases of LOS $(n=12)$

\begin{tabular}{|l|l|l|}
\hline Bacteria (n=12) & Number & $\%$ \\
\hline Coagulase Negative Staphylococci & 5 & 41.6 \\
\hline Staphylococcus aureus & 2 & 16.6 \\
\hline Acinetobacter lowffi & 2 & 16.6 \\
\hline Enterococcus spp & 1 & 8.3 \\
\hline Citrobacter freundii & 1 & 8.3 \\
\hline Pseudomonas species & 1 & 8.3 \\
\hline
\end{tabular}


In LOS the Sensitivity, Specificity, PPV, NPV and Accuracy for PCT was $66.6 \%, 50 \%$, $66.6 \%, 50 \%$ and $60 \%$. For IL-6 was $66.6 \%$, $50 \%, 66.6 \%, 50 \%$ and $60 \%$. For hs-CRP $50 \%, 75 \%, 75 \%, 50 \%$ and $60 \%$ respectively.

PCT is more sensitive and specific in EOS than IL-6 followed by hs-CRP. PCT was higher as compared to IL-6 and hs-CRP in patients with clinical evidence of EOS and even higher in those with a positive blood culture with a mean level $5.52 \mathrm{ng} / \mathrm{ml}$. Probably this was because the neonates presented to our hospital earlier within 72 hours of age. Both PCT and IL-6 are equally good markers, if patient presented later. Whereas, hs-CRP has comparatively higher specificity as compare to PCT and IL-6 in LOS.

Clinical improvement was observed more in cases of LOS $(91.66 \%)$ than EOS $(89.13 \%)$. Similarly the mortality rate was less in LOS $(2.17 \%)$ as compared to EOS (10.86\%). Probably a combination of all the three markers should be used to screen neonatal sepsis cases as all biomarkers rises at different hours of sepsis and has different half life.

\section{Ethical approval}

The study was conducted after obtaining ethical approval from the institutional ethical committee.

\section{References}

Abdollah A, et al., 2012. "Diagnostic value of simultaneous measurement of Procalcitonin, Interleukin 6 and Hs CRP in prediction of Early Onset Neonatal Sepsis." Mediterr J Hematol Infect Dis. V4(1) Stoll B.J., et al.2010 Neonatal outcomes of extremely preterm infants from the NICHD Neonatal Research Network.
Peadiatrics. 126(3): 443-56.

Alireza A, et al., 2012. Diagnostic value of simultaneous measurement of procalcitonin, IL-6 and hs-CRP in prediction of neonatal sepsis Me diter J Hematel Infect Dis.1:4(1).

Chaturvedi P, et al., 1989. Analysis of blood culture isolates from neonates of a rural hospital. Indian Pediatrics.26(5): 460-5.

Chugh K, et al., 1988. Bacteriological profile of neonatal septicemia. The Indian Journal of Pediatrics.55(6):961-5.

Edgar DM, et al., 2010. prospective study of the sensitivity, specificity and diagnostic performance of soluble intercellular adhesion molecule 1, highly sensitive C-reactive protein, soluble E-selectin and serum amyloid $A$ in the diagnosis of neonatal infection. J. BMC Pediatrics. 10(1): 22:1-16.

Fanaroff A, Martin R. 2006. Neonatal perinatal medicine. 8th ed. Mosby; 791-799.

Kayange N., et al., 2010. Predictors of positive blood culture and deaths among neonates with suspected neonatal sepsis in a tertiary hospital, MwanzaTanzania.BMCPediatr.10(1): 39-3.

MacDonald M, et al., 2005. Avery's Neonatalogy, Neonatal pathophysiology and management of the newborn. 6th ed. Philadelphia: Lippincott Williams\&Wilkins.p.12361251.

Motara F., et al., 2005. Epidemiology of neonatal sepsis at Johnnesburg hospital. South Afr. J. Epidemiol. Infect. 20(3): 90-3.

Murphy K., Weiner J. 2012. Use of leucocyte counts in evaluation of early onset neonatal sepsis. Pediatr. 31(1):16-9.

National Neonatology Forum of India 2002. Department of Pediatrics \& 
Neonatology, AIIMS, New Delhi.

Ng PC, et al., 1997. Diagnosis of late onset neonatal sepsis with cytokines, adhesion molecule, and C-reactive protein in preterm very low birth weight infants. Arch Dis Child Fetal Neonatal Ed 77:F221-227.

Shahian M, Pishva N, Kalani M. 2010. Bacterial etiology and Antibiotic sensitivity patterns of Early-Late onset Neonatal sepsis among Neoborns in Shiraz, Iran 2004-2007. 16; 35(4): 293-8.
Sharma PP, et al., 1987. Bacteriological profile of neonatal septicemia. Indian Pediatrics. 24: 1011-7.

Stoll B.J., et al., 2010. Neonatal outcomes of extremely preterm infants from the NICHD Neonatal Research Network. Peadiatrics. 126(3): 443-56.

Van den Hoogen A, et al., 2009. Long term trends in the epidemiology of neonatal sepsis and Antibiotic susceptibility of Causative Agents. Neonatalogy. 97(1):22-28.

\section{How to cite this article:}

Parul Singhal, Vichal Rastogi and Ayesha Nazar. 2019. Role of Highly Sensitive C- Reactive, Protein, Interleukin-6 and Procalcitonin in Early Diagnosis of Neonatal Sepsis. Int.J.Curr.Microbiol.App.Sci. 8(04): 245-252. doi: https://doi.org/10.20546/ijcmas.2019.804.026 